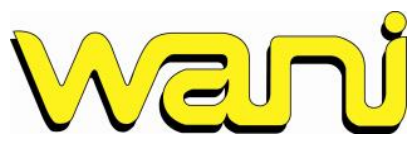

Revista del Caribe Nicaragüense

Núm. 76 | enero - junio, 2022
e-ISSN 2308-7862

Bluefields Indian \& Caribbean University (BICU)

DOI: https://doi.org/10.5377/wani.v38i76.13541 http://revistas.bicu.edu.ni/index.php/wani wani@bicu.edu.ni

\title{
Caracterización y Biometría de la Ictiofauna en la Laguna de Karata, Costa Caribe Norte Nicaragüense
}

\section{Characterization and Biometry of Ichthyofauna in the Laguna of Karata, North Caribbean Coast of Nicaragua}

\author{
iD Néstor González-Aleman ${ }^{1}$ \\ ngonzalezaleman@yahoo.es
}

(iD) Álvaro Mairena-Valdivia ${ }^{2}$

alvaro.mairena@bicu.edu.ni

Ennus Isaul Córdoba-Rivera ${ }^{3}$

ennuscordoba90@yahoo.com

Otoniel Webster-Logan ${ }^{3}$

websterlog92@gmail.com

Fecha de Recepción: 23-11-2021

Fecha de Aprobación: 09-02-2022

\section{RESUMEN}

Por la importancia biológica y ecológica que tiene la laguna de Karata para pescadores artesanales de comunidades aledañas, se completó una investigación -a través de tres muestreos por cada zona de estudio- con la finalidad evaluar el estado del recurso escama y contribuir así a la conservación y gestión del mismo. Fueron capturados un total de 692 individuos, 21 especies identificadas pertenecientes a 17 géneros, 11 familias y tres órdenes (todos de la clase Actinopterigios). El índice de diversidad fue de $\left(\mathrm{H}^{\prime}=2,21\right)$; riqueza $(\mathrm{S}=11$ familias, 17 géneros y 21 especies), y su equitatividad $(\mathrm{E}=0,72)$.

El análisis biométrico se realizó en 6 de las 21 especies identificadas, tomando como referencia las capturas mayores de 50 ejemplares de la misma especie; siendo estas las de mayor interés comercial: Bairdiella ronchus, Bagre marinus, y del género Centropomus: C. ensiferus, $C$. parallelus, $C$. pectinatus y $C$. undecimalis. A cada especie se le detalló: número de capturas, rango de edad, modelo de crecimiento (según Von Bertalanffy), factor de forma $F C$ para determinar el tipo de crecimiento y la relación longitud-peso. Se concluyó que: la población es joven; la diversidad y equitatividad, media; la riqueza, alta (debido a condiciones naturales favorables); el factor de condición $(F C)$, normal; variedades de peces con crecimiento alométrico positivo o

\footnotetext{
${ }^{1}$ Bluefields Indian \& Caribbean University, Facultad de Recursos Naturales y Medio Ambiente, Docente, Bluefields, Nicaragua.

${ }^{2}$ Bluefields Indian \& Caribbean University, Facultad de Recursos Naturales y Medio Ambiente, Director de la Escuela de Biología, Bluefields, Nicaragua.

${ }^{3}$ Bluefields Indian \& Caribbean University, Facultad de Recursos Naturales y Medio Ambiente, Licenciatura en Biología Marina, Bluefields, Nicaragua.
} 


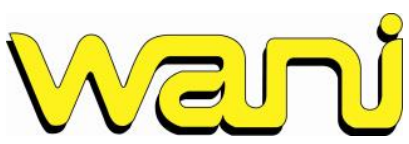

Revista del Caribe Nicaragüense

Núm. 76 | enero - junio, 2022
e-ISSN 2308-7862

Bluefields Indian \& Caribbean University (BICU)

DOI: https://doi.org/10.5377/wani.v38i76.13541

http://revistas.bicu.edu.ni/index.php/wani

wani@bicu.edu.ni

negativo, y también que el género Centropomus es el más codiciado por sus capturas e importancia comercial para la región.

Palabras clave: recurso pesquero, diversidad, biometría de forma, Karata-Nicaragua.

\section{ABSTRACT}

Due to the biological and ecological importance of the Karata lagoon for artisanal fishermen from surrounding communities, an investigation was completed -through three samplings for each study area- with the purpose of evaluating the state of the scale resource and thus contributing to the conservation and its management. A total of 692 individuals were captured, 21 identified species belonging to 17 genera, 11 families and three orders (all of the Actinopterigios class). The diversity index was $\left(\mathrm{H}^{\prime}=2.21\right)$; richness $(\mathrm{S}=11$ families, 17 genera and 21 species $)$, and its evenness $(\mathrm{E}=$ $0.72)$.

The biometric analysis was carried out in 6 of the 21 species identified, taking as reference the captures greater than 50 specimens of the same species; these being the ones of greatest commercial interest: Bairdiella ronchus, Bagre marinus, and of the Centropomus genus: $C$. ensiferus, $C$. parallelus, $C$. pectinatus and $C$. undecimalis. Each species was detailed: number of captures, age range, growth model (according to Von Bertalanffy), FC form factor to determine the type of growth and the length-weight relationship. It was concluded that: the population is young; diversity and equity, medium; richness, high (due to favorable natural conditions); the condition factor (FC), normal; varieties of fish with positive or negative allometric growth, and also that the Centropomus genus is the most coveted for its catches and commercial importance for the region.

Keywords: fishery resource, diversity, shape biometry, Karata-Nicaragua.

\section{BILA PRAHNI RA}

Tawan sirpi nani Karata lakun ka lamara nani ba mâpara naha ba baksakan plis ka kumsa kan daiwan bara dus kiamka nani sahwaya bara raya iwaya bilka kum sa, baha mihta naha pliki laki kaikan ka na sip kan dauki nata prakaya muestreo yumhpa daukan stadi takaya baikisakanka pliska nani kumi bani wina - baku natkara sip kan sins tanka pliki kaikaya inska nani sat sat bara ba nahki ra sa sapa baku natka ra kaina kahbaya bara natka yamni ra yus munaia mâta. Sut bara 692 inska alkan, baha wina 21 kiamka nani, 17 gènero, 11 familias baku sin ordenes nani 3 (Actinopterigios satka nani). Índice de diversidad ba $\left(\mathrm{H}^{\prime}=2,21\right)$; riqueza $(\mathrm{S}=11$ familia nani, 17 género nani y 21 especie nani), baku sin equitatividad $(\mathrm{E}=0,72)$.

21 inska kiamka sat sat nani alkan ba wina, 6 nani ba laki kaikan wîna paskanka ba dukiara, ani kiamka 50 inska wina purara alkan nani ba baman; baha sut wina atkaya naskaya nani ba naha nani kan: Bairdiella ronchus, Bagre marinus, y género Centropomus wina: C. ensiferus, C. parallelus, C. pectinatus bara $C$. undecimalis. Kiamka kumi bâni ra stadi munan: inska nahki pit alkan ba, nahki mâni bri ba, nahki kat pâwan ka bara mânka kulkanka ba (Von Bertalanffy wal), factor de forma FC ba yus munan kan dia sat pâwanka brisa bara yarka baku sin wirhka wal praki kaikaya dukiara. Tnata prakanka bila ba naku sa: inska nani kiamka satka nani lakun ra ba almuk apia sa, 


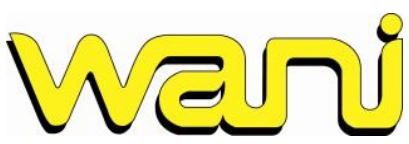

Revista del Caribe Nicaragüense

Núm. 76 | enero - junio, 2022
e-ISSN 2308-7862

Bluefields Indian \& Caribbean University (BICU)

DOI: https://doi.org/10.5377/wani.v38i76.13541 http://revistas.bicu.edu.ni/index.php/wani wani@bicu.edu.ni

diversidad bara equitatividad ba bakriki prais ra sa; riqueza ba purara sa (kan lakun ka ba bilka aiska brisa inska nani sahwaya bara pâwaya dukiara); factor de condición (FC) ba, pain sa; inska kiamka sat sat nani ba wîna pawanka yamni sa bara saura sin sa; baku sin Centropomus genero ka ba kau lihkisa kan kau ailal alkisa baku sin atkisa wan klauna baikisakanka tasbaya ra.

Baksakan bîla nani: inska ritska nani, kiamka sat sat nani, wîna paskanka sat sat nani, KarataNikarawaa.

Para citar este artículo (APA): González-Alemán, N., Mairena-Valdivia, A., Córdoba-Rivera, E. I. y Webster-Logan, O (2022). Caracterización y Biometría de la Ictiofauna de la Laguna de Karata en la Región Autónoma de la Costa Caribe Norte (RACCN). Wani(76),84-101. https://doi.org/10.5377/wani.v38i76.13541

\section{INTRODUCCIÓN}

La laguna de Karata es la tercera más grande de la Región Autónoma de la Costa Caribe Norte (RACCN), con una superficie de $33,6 \mathrm{~km}^{2}$. Se accede a ella a través del corredor fluvial que comienza en Lamlaya y se sigue por la laguna menor dividida en tres partes: hacia el noroeste remontando el río Wawa, en dirección a la laguna de Dakban; un segundo ramal atraviesa la laguna y continúa en dirección sudoeste, surcando aguas del río Wakwak; y un tercer recorrido sigue al rumbo sureste, que conduce a Karata y Wawa, terminando en la Bocana (MIKUPIA \& MARENA, 1997).

Los recursos pesqueros son una de las principales riquezas del litoral caribe nicaragüense, y sus condiciones hidro climáticas son propicias para la explotación del recurso pesquero en la laguna de Karata; las principales especies que se capturan en la zona son el robalo (Centropomus undecimalis), palometa (Eugerres plumieri), mojarra (Vieja maculicauda), barbudo (Bagre marinus), el sábalo (Megalops atlanticus) y el camarón natural (Palaemonetes sp). Estos dos últimos son predominantes en temporada de verano (Arauz, 2001; Camacho \& Gadea, 2005; Cotto S., 2001; DIPAL \& CIRH, 1997).

La pesca artesanal es la primera forma de pesca que conoció el hombre y ha sido, desde épocas remotas, la base de casi todos los asentamientos humanos en las zonas costeras. Sin embargo, esta actividad atraviesa por una etapa de crisis, agravada por la sobreexplotación y la progresiva disminución de los recursos hidrobiológicos (Camacho \& Gadea, 2005; Cervigon et al., 1992; Cotto S., 2001; Fischer et al., 1995; MIKUPIA \& MARENA, 1997).

Debido a la gran importancia biológica y ecológica que tiene esta laguna para los pescadores artesanales de las comunidades aledañas, el estudio realizado tiene como propósito identificar las diferentes especies de peces y realizar un análisis biométrico de aquella de mayor interés comercial para la zona. Esto permitirá conocer el estado actual de estas poblaciones en la laguna de Karata y así contribuir a la conservación y gestión del recurso escama. 


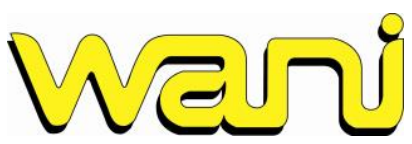

Revista del Caribe Nicaragüense

Núm. 76 | enero - junio, 2022
e-ISSN 2308-7862

Bluefields Indian \& Caribbean University (BICU)

DOI: https://doi.org/10.5377/wani.v38i76.13541 http://revistas.bicu.edu.ni/index.php/wani wani@bicu.edu.ni

Los estudios sobre la dinámica de las diferentes poblaciones acuícolas son de vital importancia para el manejo racional y sostenible (Pauly, 1983). Los estudios biológicos pesqueros (Lizama \& Ambrósio, 2002) y las evaluaciones de stock (Caballero-chávez, 2009; Caballero-Chávez, 2011; Entsua-Mensah, Osei-Abunyewa, \& Palomares, 1995) son muy importantes para el conocimiento de aspectos básicos cuantitativos, como el crecimiento en talla y peso; esta relación longitud-peso de los peces nos permite realizar mediciones de la biomasa (Froese, 2006).

El siaénido Bairdiella ronchus "Cuvier, 1830”, es una especie eurihalina con una abundancia muy elevada (Barrios, 1981)y muy explotada por los pescadores artesanales ya que representa una importante fuente de alimentación e ingresos económicos (Castro et al., 1999). Es un organismo típicamente estuarino que vive sobre fondos arenosos, crece hasta unos $35 \mathrm{~cm}$, aunque la mayoría no llega a los $30 \mathrm{~cm}$ (Dahl G., 1971).

El Ariidae Bagre marinus "Mitchill, 1815" es una especie de importancia en la zona costera (Caballero-chávez, 2009), se comercializa fresca entera, congelada y salada (Camacho \& Gadea, 2005). Su reproducción va de mayo a septiembre, con un pico de junio a agosto (Caballero-chávez, 2013; Lima et al., 2016). Esta especie se reproduce mediante incubación oral (Caballero-chávez, 2013) y presenta mortalidad alta (Mendoza-Carranza et al., 2012).

El género Centropomus se encuentra distribuido a lo largo de las aguas costeras tropicales de América (Fischer et al., 1995; Roberson \& Allen, 2006). Estos peces tienen ciclos de vida complejos con cambios ontogenéticos entre distintos entornos litorales (Vergara-Chen, 2014). Impactos sobre los hábitats han generado preocupación para el sector artesanal y la pesca deportiva (V. Caballero-Chávez, 2004; Chavez-Caballero et al., 2014). Estos peces son organismos eurihalinos, diádromos, dependientes de aguas salobres y estrechamente relacionados con los manglares estuarinos (Taylor et al., 2000). El período reproductivo varía según la especie (Andrade et al., 2013; Lorán-Núñez et al., 2012; Maldonado-García et al., 2005; Perera-Garcia et al., 2011).

\section{MATERIALES Y MÉTODOS}

\section{Descripción de la zona y tipo de estudio}

La laguna de Karata se encuentra localizada en la RACCN (Latitud de: $13^{\circ} 56^{\prime} 00^{\prime \prime} \mathrm{N}$ y Longitud de: $\left.83^{\circ} 30^{\prime} 00^{\prime \prime} \mathrm{W}\right)$, es la tercera más grande de la región $\left(33,63 \mathrm{~km}^{2}\right)$. La comunidad de Karata comprende 36,6 kilómetros cuadrados de superficie. La pesca artesanal representa el principal ingreso económica para los pobladores de las zonas aledañas. 


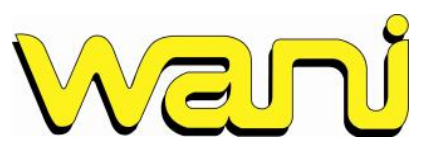

Revista del Caribe Nicaragüense

Núm. 76 | enero - junio, 2022

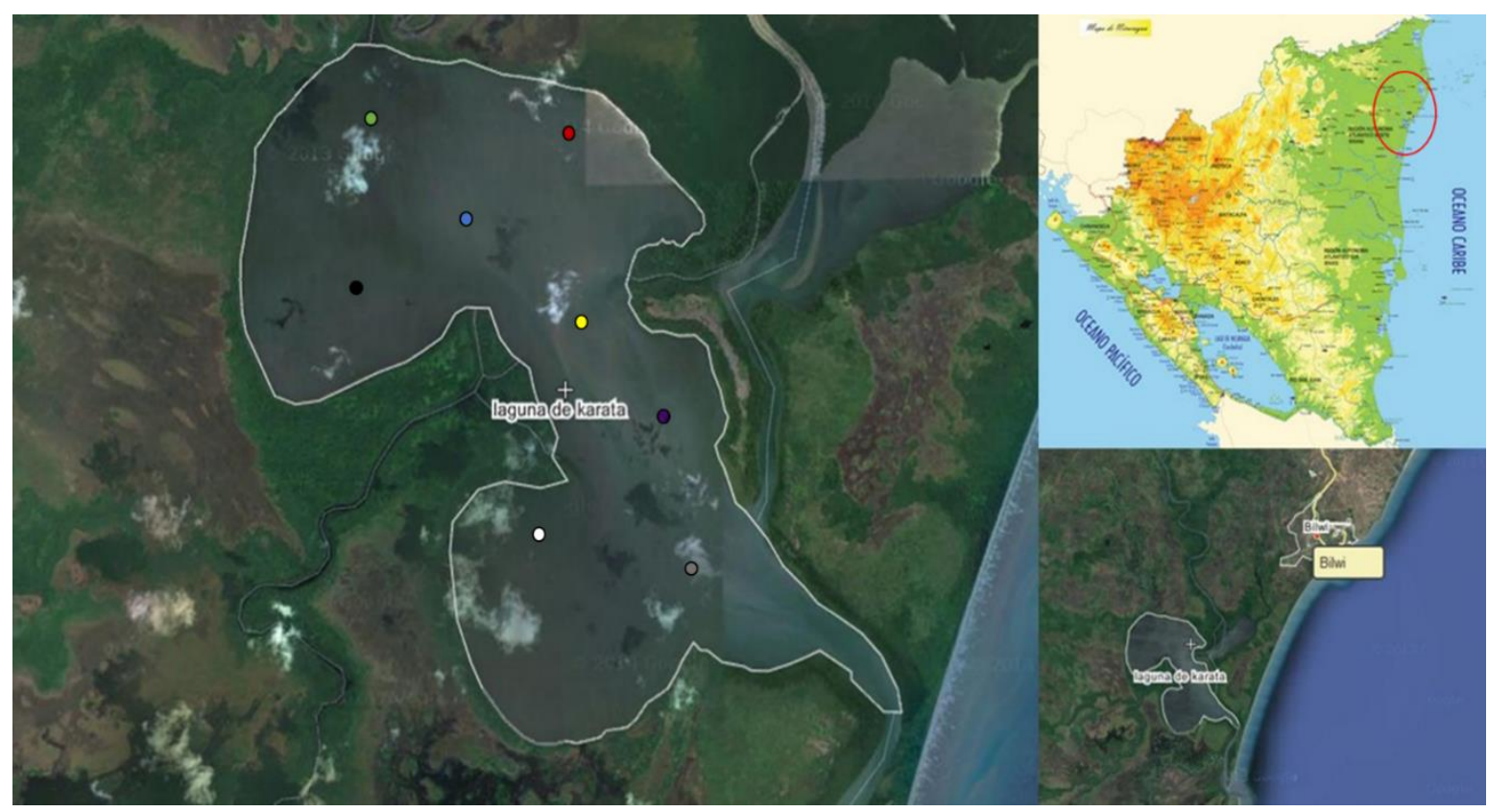

Figura 1. Mapa de ubicación de los sitios de muestreo. Zona 1. Wawa bar (gris), Zona 2. Bila tara (blanco), Zona 3. Kauhru pura (morado), Zona 4. Sita raya (amarrillo), Zona 5. Raya tara (negro), Zona 6. Yulu bila (celeste), Zona 7. Waham ta (rojo), Zona 8. Buhni ta (verde).

El estudio es de tipo descriptivo y parcialmente cuantitativo, es decir, no solo hace una descripción e identificación de las especies, sino también de sus principales parámetros poblacionales (clases de edad, población, crecimiento y relación longitud-peso). El universo de este estudio lo constituye la ictiofauna. La población la conforma la fauna íctica de la laguna de Karata y lo muestra el total de capturas realizadas durante las fases de campo, que comprenden tres viajes en un total de ocho zonas de estudio (Figura 1).

\section{Fase de campo}

\section{Primer viaje}

1) Zona 1: Wawa Bar (N 13 55 49“; W $\left.083^{\circ} 27^{\prime} 35^{\prime \prime}\right)$. Esta zona se encuentra veinte minutos hacia el sur de la comunidad de Karata, y en las cercanías de la comunidad de Wawa.

2) Zona 2: Bila Tara (N $13^{\circ} 57^{`} 73^{\prime \prime}$; W $083^{\circ} 29^{`} 88^{`}$ ). Está situada en el noreste de la comunidad de Karata.

3) Zona 3: Kauhru Pura (N $13^{\circ} 56^{`} 51^{\prime \prime}$; W $\left.083^{\circ} 28^{`} 37^{\prime \prime}\right)$. Se ubica en el lado noreste de la comunidad que va desde la segunda zona. 
Wer

Revista del Caribe Nicaragüense

Núm. 76 | enero - junio, 2022
e-ISSN 2308-7862

Bluefields Indian \& Caribbean University (BICU)

DOI: https://doi.org/10.5377/wani.v38i76.13541 http://revistas.bicu.edu.ni/index.php/wani

\section{Segundo viaje:}

1) Zona 4: Sita Raya (N $13^{\circ} 55^{`} 74^{\prime \prime}$; W $\left.083^{\circ} 27^{`} 80^{\prime \prime}\right)$. Se halla al lado norte de la comunidad que se encuentra desde la tercera zona.

2) Zona 5: Raya Tara (N 1356 44“; W $083^{\circ} 28^{`} 32^{\prime}$ ). Se sitúa al noroeste de la comunidad de Karata.

3) Zona 6: Yulu Bila (N $13^{\circ} 55^{\prime} 42^{\prime}$; W $\left.083^{\circ} 28^{`} 80^{\prime \prime}\right)$. Esta zona se encuentra al norte de Karata y se llega a ella de veinte a veinticinco minutos.

\section{Tercer viaje:}

1) Zona 7: Wahan Ta (13 $56^{`} 52^{\prime}$; W $\left.083^{\circ} 28^{`} 38^{\prime}\right)$. Se sitúa al sureste de la comunidad llegando a ella en 10 minutos.

2) Zona 8: Buhni $\mathrm{Ta}\left(\mathrm{N} 13^{\circ} 56^{`} 44^{\prime}\right.$; W $\left.083^{\circ} 28^{`} 42^{\prime \prime}\right)$. Ubicada al este de la comunidad de Karata.

Durante las capturas realizadas en cada una de las zonas de estudio se obtuvieron los principales datos biométricos, con los cuales se procedió a calcular la relación entre longitud y el peso, crecimiento de Von Bertalanffy, captura por unidad de esfuerzo (CPUE) y los índices de diversidad.

El análisis biométrico se realizó con las especies de captura mayores a cincuenta individuos; éstas resultaron ser las especies de mayor importancia para la pesca artesanal: Bairdiella ronchus, Bagre marinus, y el Genero Centropomus con cuatro especies: C. ensiferus, C. parallelus, C. pectinatus y $C$. undecimalis.

\section{Fase de laboratorio}

Para la identificación de las especies fueron utilizadas guías de campo de las especies comerciales marinas y de agua salobres (Cervigon et al., 1992; Cotto S., 2001); guía de identificación de los peces de agua dulce del lago de Nicaragua (Villa, 1982), y los estudios realizados por (Aráuz, 2001) y (González-Alemán, 2006), sobre la caracterización de las especies de peces de valor comercial de la bahía de Bluefields y la biodiversidad de peces de Mahogany con su afluente caño negro.

\section{Artes de pesca}

Para la captura de los ejemplares se utilizaron las siguientes artes de pesca: trasmallo con una luz de malla de tres pulgadas y una longitud de 100 metros (m) y 3,5 metros (m) de alto; anzuelo o línea de mano de 0,2 a 0,7 centímetros $(\mathrm{cm})$; carnadas de camarón, sardina y restos de pescado y atarraya (las dimensiones de este arte de pesca fueron de 6 pies de altura, con un radio de 6 pies y luz de maya de 1,5 centímetros $(\mathrm{cm})$. 
Wer

Revista del Caribe Nicaragüense

Núm. 76 | enero - junio, 2022
e-ISSN 2308-7862

Bluefields Indian \& Caribbean University (BICU)

DOI: https://doi.org/10.5377/wani.v38i76.13541 http://revistas.bicu.edu.ni/index.php/wani

\section{Análisis estadístico}

Para el cálculo de la captura por unidad de esfuerzo (CPUE) se utilizó la siguiente ecuación:

$$
\text { CPUE = Captura/Esfuerzo }
$$

Al igual que Martínez-Morales, José Oliva-Paterna, Verdiell-Cubedo, \& Torralva (2010), los resultados de $C P U E$ se contabilizaron por zonas de pesca, artes de pesca y el número de viajes por zonas.

El cálculo de la diversidad se realizó utilizando el índice de Shannon-Weaver,

$$
H^{\prime}=-\sum_{i=1}^{S}\left(p_{i} * \log _{2} p_{i}\right)
$$

donde $\mathrm{p}_{\mathrm{i}}$ es la proporción real de individuos $\left(\mathrm{N}_{\mathrm{i}} / \mathrm{N}\right)$ de la especie i y $\mathrm{S}$ es el número total de especies observadas (Shannon \& Weaver, 1949; Spellerberg \& Fedor, 2003).

La riqueza específica es un concepto simple de interpretar: se relaciona con el número de especies presentes en la comunidad. Entonces, puede parecer que un índice apropiado para caracterizar la riqueza de especies de una comunidad sea el 'número total de especies' (S) y (n) número de individuos observados que se incrementa con el tamaño de la muestra (Margalef, 1958).

$$
R_{1}=\frac{\mathrm{S}-1}{\operatorname{Ln}(n)}
$$

La equitatividad se calculó utilizando la fórmula de Hurlbert (1970), la cual establece que si todas las especies en una muestra presentan la misma abundancia, el índice usado para medir la equitatividad debería ser máximo y, por lo tanto, debería decrecer tendiendo a cero a medida que la abundancia relativa se haga menos equitativa.

$$
E=\frac{\mathrm{D}-D_{\min }}{D_{\max }-D_{\min }}
$$

Donde: $\mathrm{D}=$ índice de diversidad, $\mathrm{D}_{\min }=$ valor mínimo de $\mathrm{D}$ y $\mathrm{D}_{\max }=$ valor máximo de $\mathrm{D}$

El análisis del crecimiento en función al tiempo de vida se realizó utilizando el modelo exponencial de Von Bertalanffy (Ricker, 1975).

$$
\mathrm{L}(\mathrm{t})=\mathrm{L}_{\max }\left(1-\mathrm{e}^{-\mathrm{k}(\mathrm{t}-\mathrm{t})}\right)
$$

Donde: $\mathrm{L}(\mathrm{t})=$ Longitud teórica a la edad $\mathrm{t}, \mathrm{L}_{\max }=$ Longitud asintótica, $\mathrm{K}=$ Coeficiente de crecimiento, $\mathrm{t}=$ Edad del individuo en años, $\mathrm{t}_{\mathrm{o}}=$ Edad teórica del individuo a la longitud cero. 


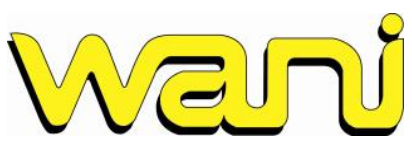

Revista del Caribe Nicaragüense

Núm. 76 | enero - junio, 2022
e-ISSN 2308-7862

Bluefields Indian \& Caribbean University (BICU)

DOI: https://doi.org/10.5377/wani.v38i76.13541 http://revistas.bicu.edu.ni/index.php/wani wani@bicu.edu.ni

Algunos parámetros cualitativos y cuantitativos de estas poblaciones de peces fueron calculados empleando el programa Dimp 1.0 (Sanz-Ronda et al., 2013), con el cual se obtuvo: clases de edad, frecuencias por clases, longitud y peso medio, densidad poblacional y biomasa.

El Factor de condición o índice de Fulton (K), de acuerdo a Froese (2006), Fulton (1904)y Granado (1996), estima las modificaciones temporales del buen estado de los peces. Este índice varía según las especies, morfología, sexo, edad, estado reproductivo de acuerdo con la madurez gonadal, época del año y el ambiente acuático.

$$
K=100 * W / L F^{3}
$$

Donde; $W$ : peso del pez en gramos, y $L F$ : longitud del pez en mm.

Finalmente, la relación entre $W$ y $L$ se calculó a través del modelo potencial ecuación $W=a *$ $L F^{b}$, donde $W=$ peso total $(\mathrm{g}), L F=$ longitud furcal $(\mathrm{cm}) a$ y $b=$ son coeficientes (Froese, 2006; Le Cren, 1951), siendo $b$ la constante de alometría y en condiciones naturales oscila entre 2,5 y 4 (Granado, 1996; Le Cren, 1951).

Debido a que la longitud es una magnitud lineal y el peso es igual al cubo de la talla, si un individuo mantiene su forma al crecer, entonces el crecimiento es isométrico $(b=3)$. Cuando $b>3$, los individuos de mayor talla han incrementado su peso en mayor proporción que su longitud, presentando crecimiento alométrico positivo. En cambio, cuando $b<3$, los individuos incrementan preferencialmente su longitud relativa más que su peso. Se consideraron especies de crecimiento isométrico las que fluctuaron dentro de los valores $b=2,5$ y $b=3,5$ (Froese, 2006).

\section{RESULTADOS Y DISCUSIÓN}

\section{Diversidad, Riqueza y Equitatividad Taxonómica}

El resultado de Diversidad $\left(\mathrm{H}^{\prime}=2,21\right)$ obtenido se considera media y aunque la riqueza es alta ( $\mathrm{S}$ = 11 Familia, 17 Género y 21 Especie), su equitatividad es baja $(\mathrm{E}=0,72)$, ya que presenta una distribución desigual en cuanto a la abundancia o número de individuos por especies (Ver anexo Tabla 2). En ecosistemas similares, Arauz (2001) encuentra valores superiores de las mismas variables. Según González-Alemán (2006), valores superiores a tres significan diversidad alta; valores entre uno y tres, diversidad media, y, los inferiores a uno, diversidad baja.

Durante las tres jornadas de pesca, el $95.81 \%$ de las capturas fuero realizadas con red agallera, $3.32 \%$ con líneas de manos o anzuelos, y solamente un $0.86 \%$ fue capturado con atarraya, resultando esta última la menos eficiente (Tabla 1). 


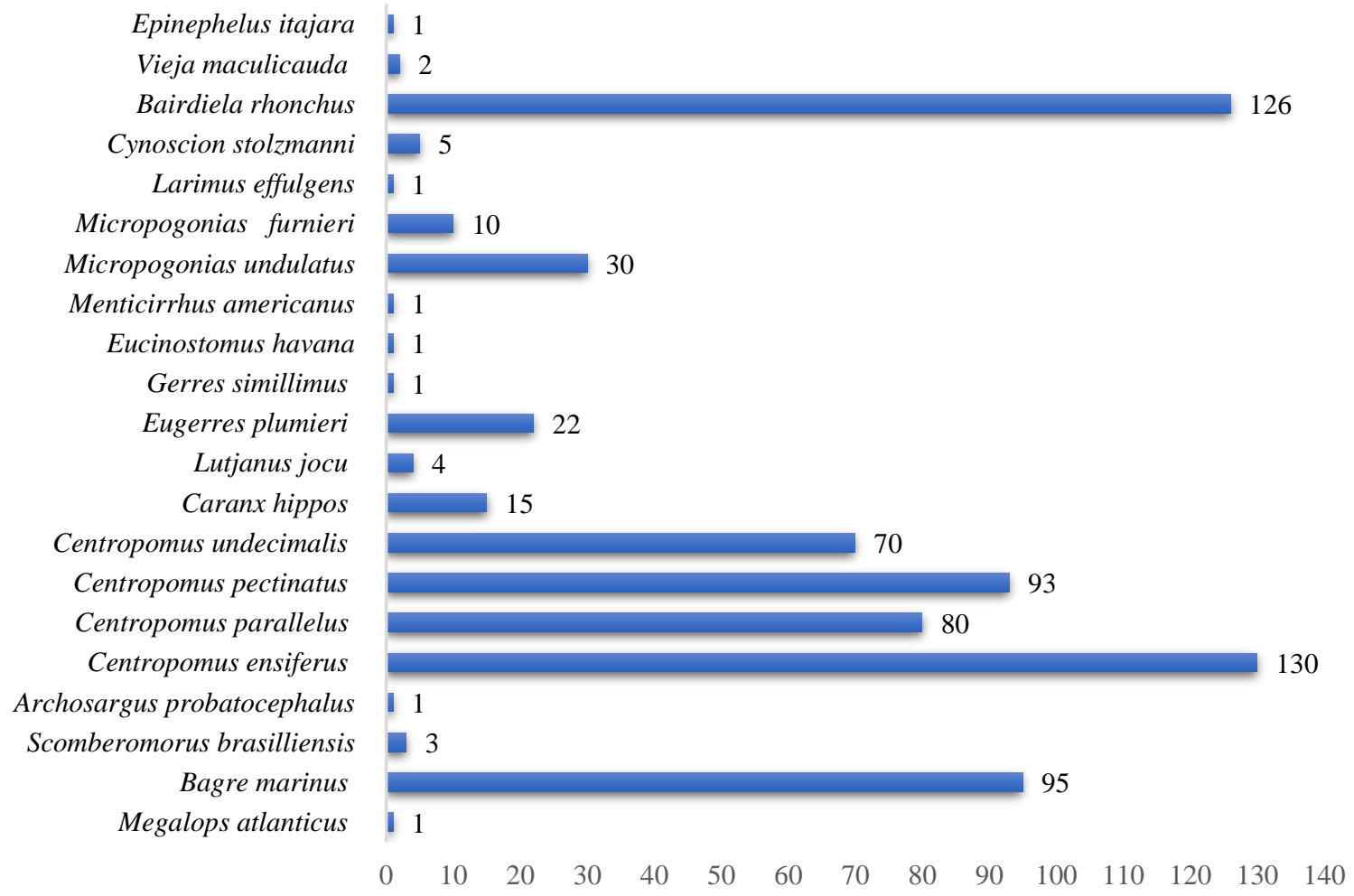

Figura 2. Capturas por unidad de esfuerzo (CPUE) realizadas por especie

\section{Captura por unidad de esfuerzo (CPUE)}

El Orden Perciformes presentó nueve familias de las 11 reportadas; la Familia Sciaenidae presentó cinco géneros de los 17 encontrados, y el Genero Centropomus presentó el mayor número de especies: cuatro de las 21 identificadas.

Las capturas de mayor importancia pertenecen al género Centropomus con un total de 373 individuos capturados (53,9\% de las capturas totales), 130 de la especie $C$. undecimalis, 80 de $C$. ensiferus, 93 de $C$. parallelus y 70 de $C$. undecimalis. Comercialmente, este género es uno de los más importantes, en la región y representa el sustento para muchas de las comunidades indígenas que se ubican en sus riberas (Chavez-Caballero et al., 2014). Estos datos coinciden con un estudio realizado por Brenes \& Escoto (1988), quienes encontraron que el aporte porcentual de la producción por laguna es: Karata 46,6 \%, Bihmona $29 \%$, Pahara 12,2 \%, Wuohta 5,6 \%, y otros ríos y lagunas $4,6 \%$.

Uno de los hallazgos más importantes fue la captura de un ejemplar del mero guasa o guato Epinephelus itajara (Liechtenstein, 1822), el más grande de los serránidos de las aguas tropicales americanas y pueden alcanzar un peso de hasta $455 \mathrm{~kg}$ (Robins, C. R., Ray, G. C., Douglass, J., Freund, R., National Audubon Society, 1986). Se encuentra desde la costa Este de la Florida, el 


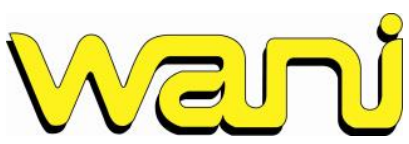

Revista del Caribe Nicaragüense

Núm. 76 | enero - junio, 2022
e-ISSN 2308-7862

Bluefields Indian \& Caribbean University (BICU)

DOI: https://doi.org/10.5377/wani.v38i76.13541 http://revistas.bicu.edu.ni/index.php/wani wani@bicu.edu.ni

Golfo de México y el Mar Caribe, hasta el Brasil, incluyendo también el océano Pacífico, desde Costa Rica hasta el Perú (Smith, 1971). Los ejemplares pequeños se encuentran principalmente en áreas estuarinas de poca profundidad, entre las raíces de mangle y substrato fangoso, desde aguas salobres frente a las desembocaduras de los ríos, hasta fondos coralinos en áreas insulares oceánicas (Cervigón, 1991).

Esta especie, Epinephelus itajara (Liechtenstein, 1822) se encuentra en la lista roja del 2006 de la Unión Mundial para la Naturaleza (UICN) en peligro crítico (CR), mientras que en el CITES (Convención sobre el Comercio Internacional de Especies Amenazadas de Fauna y Flora Silvestres) no está listado.

El género Bagre, especie Bagre marinus, representó un 13,7\% de las capturas, con 95 individuos pertenecientes al Orden Siluriformes, Familia Ariidae. Finalmente, el género Bairdiella, especie Bairdiella ronchus presento un 18,2\% de las capturas, con 126 individuos del Orden Perciformes, Familia Sciaenidae.

Tabla 1. Descripción de las Capturas por unidad de esfuerzo (CPUE), de acuerdo con las jornadas de pesca, estación y zonas.

Número de individuos capturados por Jornadas de pesca y estación

\begin{tabular}{|c|c|c|c|c|c|c|c|c|c|}
\hline \multirow{3}{*}{ Arte de pesca } & \multicolumn{3}{|c|}{$\begin{array}{c}\text { finales de } \\
\text { invierno (octubre } \\
\text { 2013) }\end{array}$} & \multicolumn{3}{|c|}{$\begin{array}{c}\text { Verano (febrero } \\
\text { 2014) }\end{array}$} & \multicolumn{2}{|c|}{$\begin{array}{l}\text { Comienzo de } \\
\text { Invierno } \\
\text { (mayo 2014) }\end{array}$} & \multirow{3}{*}{$\begin{array}{c}\text { CAPTURA } \\
\text { TOTAL }\end{array}$} \\
\hline & \multicolumn{3}{|c|}{ 1era. Jornada } & \multicolumn{3}{|c|}{ 2da. Jornada } & \multicolumn{2}{|c|}{ 3ra. Jornada } & \\
\hline & $\mathbf{Z 1 *}$ & $\mathbf{Z 2 *}$ & $\mathbf{Z 3}^{*}$ & $\mathbf{Z 4 *}$ & $\mathbf{Z 5}^{*}$ & $\mathbf{Z 6}^{*}$ & $\mathbf{Z 7 *}$ & Z8* & \\
\hline Anzuelo o Línea de Mano & 4 & 2 & 4 & 2 & 5 & 2 & 3 & 1 & 23 \\
\hline Red Agallera & 166 & 131 & 88 & 100 & 63 & 33 & 58 & 24 & 663 \\
\hline Tarraya & 2 & 0 & 1 & 0 & 1 & 0 & 2 & 0 & 6 \\
\hline $\begin{array}{c}\text { INDIVIDUOS } \\
\text { CAPTURADOS POR } \\
\text { ZONA }\end{array}$ & 172 & 133 & 93 & 102 & 69 & 35 & 63 & 25 & 692 \\
\hline $\begin{array}{c}\text { INDIVIDUOS } \\
\text { CAPTURADOS POR } \\
\text { JORNADA }\end{array}$ & & 398 & & & 206 & & & & \\
\hline
\end{tabular}




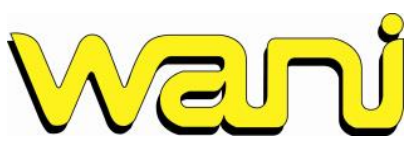

Revista del Caribe Nicaragüense

Núm. 76 | enero - junio, 2022
e-ISSN 2308-7862

Bluefields Indian \& Caribbean University (BICU)

DOI: https://doi.org/10.5377/wani.v38i76.13541

http://revistas.bicu.edu.ni/index.php/wani

wani@bicu.edu.ni

\section{Análisis biométrico}

El crecimiento de la población en Bairdiella ronchus está condicionado por el número de individuos en la clase de edad 2+ y 3+, Se contabilizó un total de 101 individuos entre los dieciocho y ventitrés centímetros de longitud. Según Castro et al (1999), la talla promedio de madurez es de 15,8 cm en las hembras, y de 15,4 cm en los machos Yáñez-Arancibia et al. (1985) encontró que la alta fecundidad está relacionada con la talla. El factor de condición se considera alto, en comparación con lo encontrado por Castro et al (1999), y puede estar relacionado con el desarrollo de los órganos sexuales (Mendo et al., 1988), aunque, para Wootton (1990) el factor de condición está limitado por la disponibilidad, cantidad y calidad de alimento presente en el medio. En cuanto a la relación longitud-peso, esta especie presenta un crecimiento alométrico negativo (b < 3); el modelo explica $75.3 \%$ de esa relación.

En Bagre marinus el crecimiento está condicionado por la clase de edad 1+ y 2+. Según Dahl G. (1971), esta especie puede llegar a crecer hasta $60 \mathrm{~cm}$, en comparación con $B$. ronchus que alcanza los $35 \mathrm{~cm}$. La talla de la primera madurez en las hembras es de 35,8 cm y en los machos es de 25,1 cm según Caballero-chávez (2013). El factor de condición en esta especie está directamente relacionado con el crecimiento dorsoventral que tiene esta especie durante la época de reproducción (Segura-Berttolini \& Mendoza-Carranza, 2013). El tamaño en esta especie es de vital importancia para la incubación de los huevos (Mendoza-Carranza et al., 2012), existiendo una relación lineal positiva entre la talla de la hembra y el número de huevos próximos al desove (Pinheiro et al., 2006), además produce un número reducido de huevos y una alta supervivencia de su progenie (Winemiller \& Rose, 1992). Esta especie presenta un crecimiento alométrico positivo (b $\geq 3$ ); el modelo de la relación longitud-peso sólo explica el $45 \%$.

En $C$. ensiferus, el crecimiento de la población está condicionado por la clase de edad 2+; en $C$. parallelus, por las clases de edad $1+\mathrm{y} 2+$; en $C$. pectinatus, no hay preferencias de clases, pues, según Camacho \& Gadea (2005) y Vergara-Chen (2014), el crecimiento de esta población es homogéneo y está relacionado con los periodos de desove. En C. undecimalis, el crecimiento de la población está condicionado por la clase de edad 1+. El Factor de condición para este género está relacionado con la época de reproducción (Caballero-chávez, 2009; Caballero-Chavez et al., 1995; Vequi Caballero-Chávez, 2011; Camacho \& Gadea, 2005; Chavez-Caballero et al., 2014; Gadea, 2002). Centropomus pectinatus y $C$. undecimalis presentan un crecimiento alométrico negativo (b $<2,5$ ), mientras que $C$. parallelus y $C$. ensiferus evidencian un crecimiento alométrico positivo (b $\geq 2,5 \mathrm{y} \mathrm{b} \geq 3,5$ ). La talla de la primera madurez sexual en hembras de $C$. parallelus es de $15 \mathrm{~cm} \mathrm{y}$ $15,4 \mathrm{~cm}$ en machos; en $C$. undecimalis, las hembras maduran a los $38,8 \mathrm{~cm}$ y los machos a los 34,3 cm (Alvarado, 2004); en C. ensiferus, la talla de la primera maduración en hembras se da a los 26 


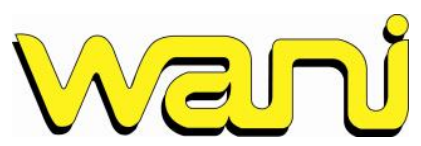

Revista del Caribe Nicaragüense

Núm. 76 | enero - junio, 2022
e-ISSN 2308-7862

Bluefields Indian \& Caribbean University (BICU)

DOI: https://doi.org/10.5377/wani.v38i76.13541 http://revistas.bicu.edu.ni/index.php/wani

cm y 21,5 cm en machos (Gassman et al., 2017); en C. pectinatus, el 50\% de la población alcanza la madurez sexual cuando logra una longitud de $24 \mathrm{~cm}$ (Freitas \& Abilhoa, 2017).

Figura 3. Relación longitud-peso de Bairdiella ronchus (a) y Bagre marinus (b) en la laguna de Karata durante el periodo 2013-2014.

a

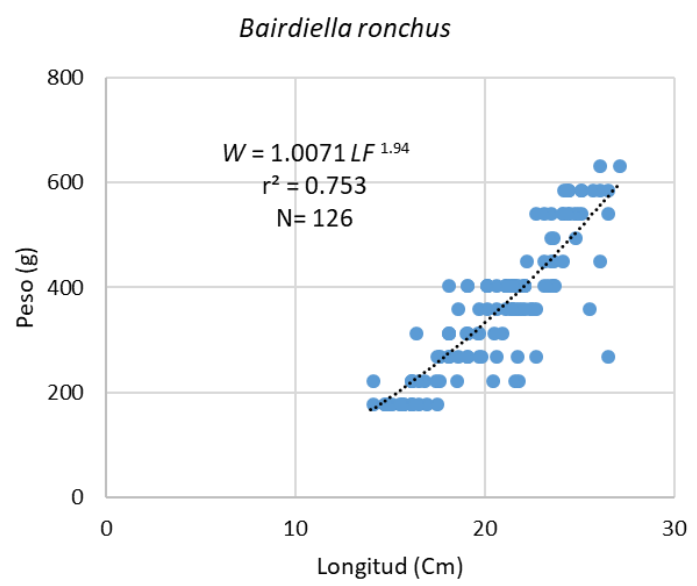

b

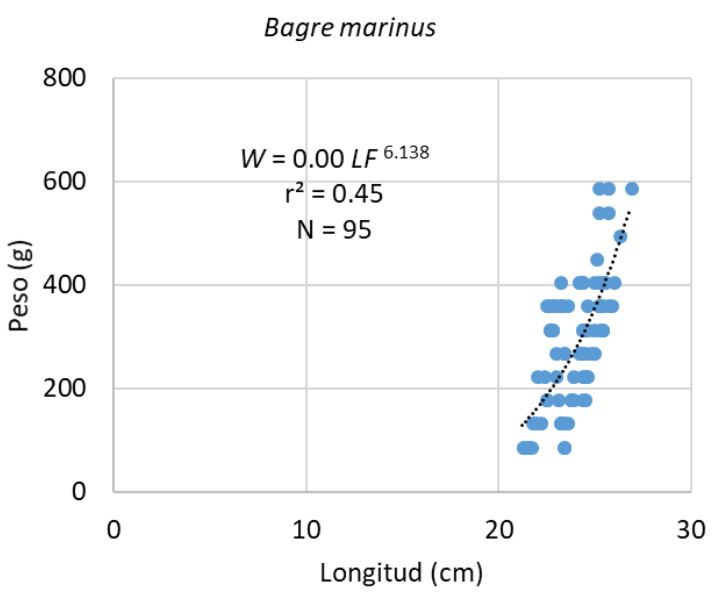

Figura 4. Relación Longitud-Peso en el género Centropomus durante el periodo 2013-2014. C. ensiferus (a), C. pectinatus (b), C. parallelus (c) y C. undecimalis (d).
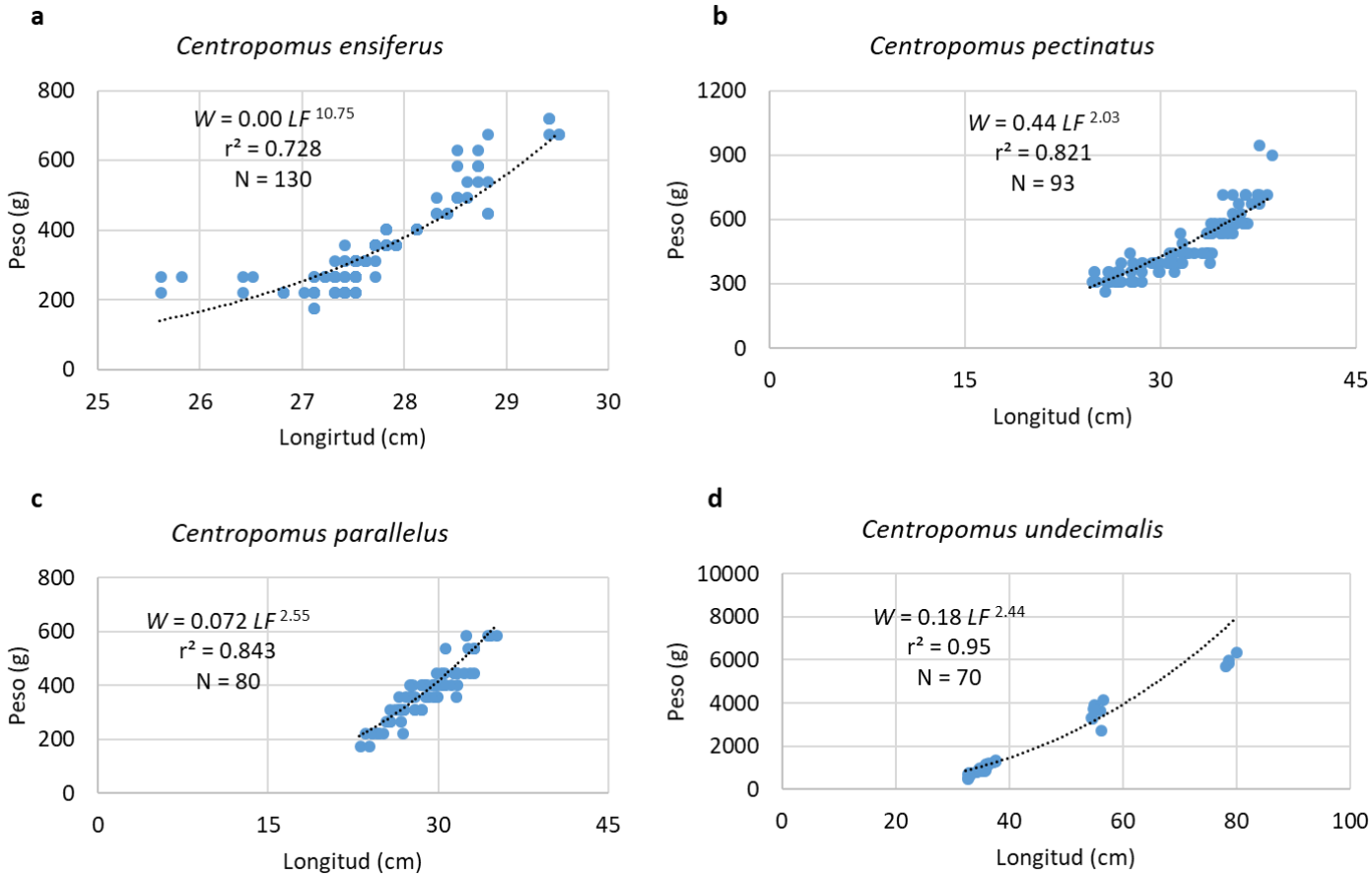

Todas las especies del género Centropomus presentan una alta fecundidad (Chavez-Caballero et al., 2014). Los resultado muestra un valor alto del Factor de condición, el cual puede estar 


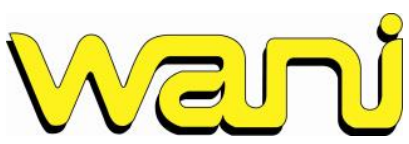

Revista del Caribe Nicaragüense

Núm. 76 | enero - junio, 2022
e-ISSN 2308-7862

Bluefields Indian \& Caribbean University (BICU)

DOI: https://doi.org/10.5377/wani.v38i76.13541 http://revistas.bicu.edu.ni/index.php/wani wani@bicu.edu.ni

asociado a los periodos de reproducción (Froese, 2006), aunque, para Wootton (1990), el factor de condición está asociado a la cantidad y calidad del alimento.

Tabla 2. Resultado biométrico de las seis especies de mayor interés comercial en la laguna de Karata.

\begin{tabular}{|c|c|c|c|c|c|c|}
\hline Variable & $\begin{array}{c}\text { Bairdiella } \\
\text { ronchus } \\
\text { (Cuvier, } \\
\text { 1830) }\end{array}$ & $\begin{array}{c}\text { Bagre } \\
\text { marinus } \\
\text { (Mitchill, } \\
\text { 1815) }\end{array}$ & $\begin{array}{l}\text { C. ensiferus } \\
\text { (Poey, 1860) }\end{array}$ & $\begin{array}{l}\text { C. pectinatus } \\
\text { (Poey, 1960) }\end{array}$ & $\begin{array}{l}\text { C. parallelus } \\
\text { (Poey, 1960) }\end{array}$ & $\begin{array}{l}\text { C. undecimalis } \\
\text { (Bloch, 1792) }\end{array}$ \\
\hline $\begin{array}{ll}\text { Clase } & \text { de } \\
\text { edad } & \end{array}$ & $\begin{array}{l}5(0+, 1+, 2+, \\
3+\mathrm{y} 4+)\end{array}$ & $\begin{array}{l}4(0+, 1+, 2+ \\
\text { y } 3+)\end{array}$ & $\begin{array}{l}5(0+, 1+, 2+, \\
3+\mathrm{y} 4+)\end{array}$ & $\begin{array}{l}4(0+, 1+, 2+y \\
3+)\end{array}$ & $\begin{array}{l}4(0+, 1+, 2+ \\
\text { y } 3+)\end{array}$ & $\begin{array}{l}6(0+, 1+, 2+, \\
3+, 4+\mathrm{y} 5+)\end{array}$ \\
\hline $\begin{array}{l}\text { Peso } \\
\text { promedio }\end{array}$ & $\begin{array}{l}0.8 \quad l b \\
(368.27 \mathrm{~g})\end{array}$ & $\begin{array}{l}1.04 \quad \mathrm{lb} \\
(471.734 \mathrm{~g})\end{array}$ & $\begin{array}{l}1.2 \quad l b \\
(544,308 \text { gr })\end{array}$ & $\begin{array}{l}1 \mathrm{lb} \quad(453,59 \\
\mathrm{gr})\end{array}$ & $\begin{array}{l}0,9 \quad \mathrm{lb} \\
(408,231 \mathrm{gr})\end{array}$ & $\begin{array}{l}2,3 \quad l b \\
(1043,247 \mathrm{gr})\end{array}$ \\
\hline $\begin{array}{l}\text { Longitud } \\
\text { promedio }\end{array}$ & $20.7 \mathrm{~cm}$ & $22.6 \mathrm{~cm}$ & $31.6 \mathrm{~cm}$ & $31,3 \mathrm{~cm}$ & $29,3 \mathrm{~cm}$ & $42,4 \mathrm{~cm}$ \\
\hline $\begin{array}{l}\text { Factor de } \\
\text { condición } \\
\text { (FC) }\end{array}$ & $0.0388 \mathrm{~g} / \mathrm{cm}^{3}$ & $0.0213 \mathrm{~g} / \mathrm{cm}^{3}$ & $\begin{array}{l}\mathrm{FC}=0.0165 \\
\mathrm{gr} / \mathrm{cm}^{3}\end{array}$ & $\begin{array}{l}\mathrm{FC}=0.0147 \\
\mathrm{gr} / \mathrm{cm}^{3}\end{array}$ & $\begin{array}{l}\mathrm{FC}=0.0157 \\
\mathrm{gr} / \mathrm{cm}^{3}\end{array}$ & $\begin{array}{l}\mathrm{FC}=0.0200 \\
\mathrm{gr} / \mathrm{cm}^{3}\end{array}$ \\
\hline
\end{tabular}

Modelo de crecimiento de Von Bertalanffy

$$
L(t)=\operatorname{Linf}-\operatorname{Linf} e^{-k(t-t o)}
$$

$\begin{array}{lllllll}\text { Crecimiento } & * \operatorname{Linf}=35 & * \operatorname{Linf}=60 & * \operatorname{Linf}=34 & * \operatorname{Linf}=56 & * \operatorname{Linf}=58 & * \operatorname{Linf}=125 \\ & \mathrm{~K}=0.220 & \mathrm{~K}=0.046 & \mathrm{~K}=0.149 & \mathrm{~K}=0.145 & \mathrm{~K}=0.099 & \mathrm{~K}=0.166 \\ & \text { To }=-1.914 & \mathrm{To}=-9.58 & \text { To }=-9.310 & \text { To }=-4.225 & \text { To }=-5.559 & \text { To }=-0.810 \\ & \mathrm{r}^{2}=0.953 & \mathrm{r}^{2}=0.996 & \mathrm{r}^{2}=0.990 & \mathrm{r}^{2}=0.997 & \mathrm{r}^{2}=0.998 & \mathrm{r}^{2}=0.982\end{array}$

\begin{tabular}{|c|c|c|c|c|c|c|}
\hline \multicolumn{7}{|c|}{$\begin{array}{l}\text { Relación Longitud-Peso } \\
\qquad W=a * L F^{b}\end{array}$} \\
\hline Longitud- & $\mathrm{a}=1.0059$ & $\mathrm{a}=0.00$ & $a=0.00$ & $\mathrm{a}=0.44$ & $a=0.072$ & $a=0.18$ \\
\hline Peso & $* \mathrm{~b}=1.94$ & $* \mathrm{~b}=6.14$ & $* \mathrm{~b}=10.75$ & $* \mathrm{~b}=2.03$ & $* \mathrm{~b}=2.55$ & $* \mathrm{~b}=2.44$ \\
\hline
\end{tabular}

*Ling = a la longitud máxima que puede alcanzar esa especie.

$* \mathrm{~b}=\mathrm{a}$ la constante de alometría.

\section{CONCLUSIONES}

La diversidad que se obtuvo durante las jornadas se considera media, al igual que su equitatividad, con excepción de la riqueza considerada alta, debido a las condiciones naturales del ecosistema estudiado. El género Centropomus es el de mayor importancia no solo por sus capturas, sino por la importancia comercial para la región.

Las clases de edades encontradas en el estudio corresponden a una población joven; esto se puede verificar a través de las longitudes y pesos medios registrados durante el estudio. Esto se debe a que se trata de una población bastante explotada por la pesca artesanal.

El factor de condición $(F C)$ se considera normal, principalmente en poblaciones que están en época de reproducción. Esto se ve reflejado en el crecimiento de la población ajustado al modelo de Von Bertalanffy el cual goza de un alto grado de confiabilidad: por encima del $95 \%$. 


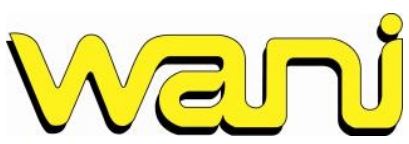

Revista del Caribe Nicaragüense

Núm. 76 | enero - junio, 2022
e-ISSN 2308-7862

Bluefields Indian \& Caribbean University (BICU)

DOI: https://doi.org/10.5377/wani.v38i76.13541 http://revistas.bicu.edu.ni/index.php/wani wani@bicu.edu.ni

Existe una relación positiva muy alta que se puede observar a través del incremento del peso en cada uno de los individuos analizados, a medida que éstos crecen en longitud. El modelo Potencial $\left(W=\mathrm{a} L F^{\mathrm{b}}\right.$ ) el cual, a través de la relación longitud-peso, nos dice que Bagre marinus, C. ensiferus y $C$. parallelus tienen un crecimiento alométrico positivo; en cambio, Bairdiella ronchus, $C$. pectinatus y $C$. undecimalis presentan un crecimiento alométrico negativo.

\section{CONFLICTO DE INTERESES}

Los autores declaran no tener conflicto de intereses.

\section{AGRADECIMIENTO}

A la Bluefields Indian \& Caribbean University (BICU), por los fondos destinados a los proyectos de Investigación del Departamento de Investigación de esta Universidad, y a los pescadores artesanales de las comunidades aledañas a la zona de estudio.

\section{DEDICATORIA}

A la memoria de la Lucia Dávila, PhD. (q.e.p.d.) quien en vida fue la Directora de Investigación de BICU. "Dejaste una huella para la eternidad; no sabemos hasta donde llegará tu influencia" (Henry Adams).

\section{REFERENCIAS}

Alvarado, L. C. P. (2004). La ictiofauna del Refugio de Vida Silvestre Bocas del Polochic y la cuenca del lago de Izabal : composición, distribución y ecología.

Andrade, H., Santos, J., \& Taylor, R. (2013). Life-history traits of the common snook Centropomus undecimalis in a Caribbean estuary and large-scale biogeographic patterns relevant to management. Journal of Fish Biology, 82(6), 1951-1974.

Arauz, J. (2001). La caracterización de las especies de peces de valor comercial de la Bahía de Bluefields y su alrededor. DIPAL, 2001.

Barrios, D. M. (1981). Estudio bioecológico de las capturas de peces con redes agalleras en la Ciénaga Grande de Santa Marta. Tesis de Licenciatura, Universidad Na-cional de Colombia, Bogotá, Colombia.

Brenes, \& Escoto. (1988). Comportamiento de los Desembarques de escamas provenientes de las lagunas costeras de la RAAN. CIRH. Managua, Nicaragua.

Caballero-chávez, V. (2009). Pesquería de robalo blanco Centropomus undecimalis en Campeche. Ciencia pesquera, 17(2), 77-86.

Caballero-chávez, V. (2013). Madurez y reproducción de bagre bandera Bagre marinus en el sudeste de Campeche. Ciencia pesquera, 21(2), 13-19.

Caballero-Chávez, V. (2004). Evaluación de la pesquería ribereña de robalo blanco Centropomus undecimalis y bandera Bagre marinus, en el sur de Campeche.

Caballero-Chavez, V., L.F. Peña-Durán, J.F. Echavarría-Vera, O. Jiménez-Morales, \& J.A. Zamora. (1995). 


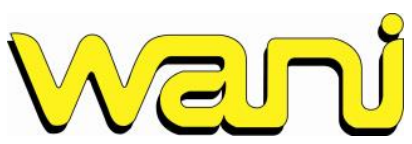

Revista del Caribe Nicaragüense

Núm. 76 | enero - junio, 2022
e-ISSN 2308-7862

Bluefields Indian \& Caribbean University (BICU)

DOI: https://doi.org/10.5377/wani.v38i76.13541 http://revistas.bicu.edu.ni/index.php/wani wani@bicu.edu.ni

Contribución al conocimiento de la biología del robalo blanco (Centropomus undecimalis Bloch, 1792). Informe de Investigación (documento interno). CRIP - Ciudad del Carmen. Instituto Nacional de la Pesca. México. 64p.

Caballero-Chávez, Vequi. (2011). Reproducción y fecundidad del robalo blanco (Centropomus undecimalis) en el suroeste de Campeche. Ciencia Pesquera, 19(1), 35-46.

Camacho, J. J., \& Gadea, V. (2005). Estudio técnico científico del róbalo en Río San Juan y el Gran Lago de Nicaragua.

Castro, L. T., Santos-Martinez, A., \& Acero, A. (1999). Reproduccion de Bairdiella ronchus (Pisces: Sciaenidae) en la Cienaga Grande de Santa Marta, Caribe Colombiano. Revista Biologica Tropical, 47(3), 553-559.

Cervigón, F. (1991). Los peces marinos de Venezuela. Fundación Científica Los Roques.

Cervigon, F., Cipriani, R., Fischer, W., Garibaldi, L., Hendrickx, H., Lemus, A. J., Marquez, R., Poutiers, J. M., Robaina, G., \& Rodriguez, B. (1992). Guia de campo de las especies comerciales marinas y de agua salobres de la costa septentrional de sur america. Preparado con el financiamiento de la Comisión de Comunidades Europeas y de NORAD. Roma, FAO, 513 pp. (p. 577).

Chavez-Caballero, V., Loran-Nuñez, R. M., Gomez-Ortiz, M. G., Garduño-Dionate, M., Martinez-Isunza, F. R., \& Wakida-Kusunoki, A. T. (2014). Robalo del Golfo de Mexico: Centropomus undecimalis, Centropomus poeyi y Centropomus parallelus. En Instituto Nacional de Pesca.

Cotto S., A. (2001). Guía de identificación de peces marinos del mar Caribe de Nicaragua. Proyecto para el Desarrollo Integral de la Pesca Artesanal en la Región Autónoma del Atlántico Sur, Nicaragua (DIPAL). Proyecto DIPAL II, 2001.

Dahl G. (1971). Los Peces del Norte de Colombia. Ministerio de Agricultura, Instituto de Desarrollo de los Recursos Naturales Renovables INDERENA. Bogotá, Colombia.

DIPAL, \& CIRH. (1997). Plan de Manejo Integral para los recursos Hidrobiológicos de la Cuenca de Laguna de Perlas y la Desembocadura del Río Grande. Laguna de Perlas, Nicaragua. 39 pp.

Entsua-Mensah, M., Osei-Abunyewa, A., \& Palomares, M. L. D. (1995). Length-weight relationships of fishes from tributaries of the Volta River, Ghana: part 1 analysis of pooled data sets. Box 38, Achimota (Ghana), M L D (Institute of Aquatic Biology. ICLARM [International Center for Living Aquatic Resources Management] Quarterly, 18(1), 36-38.

Fischer, W., Krupp, F., Schneider, W., Sommer, C., Carpenter, K. E., \& Neim, V. H. (1995). Guía FAO para la identificación de especies para los fines de la pesca. Pacífico centro-oriental. Plantas e inveertebrados. En Roma, FAO. 1995. Vol. I: 1-646 p.

Freitas, M. O., \& Abilhoa, V. (2017). Reproductive biology of the tarpon snook Centropomus pectinatus (Perciformes, Centropomidae) in estuarine waters in the south-western Atlantic. Journal of fish biology, 91(2), 686-694.

Froese, R. (2006). Cube law, condition factor and weight-length relationships: History, meta-analysis and recommendations. Journal of Applied Ichthyology, 22(4), 241-253.

Fulton, T. W. (1904). The rate of growth of fishes. 22nd Annual Report. Fishery Board of Scotland, 3, 141241. 


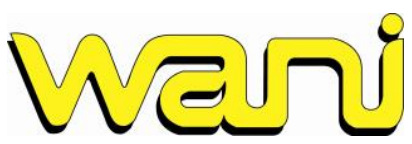

Revista del Caribe Nicaragüense

Núm. 76 | enero - junio, 2022
e-ISSN 2308-7862

Bluefields Indian \& Caribbean University (BICU)

DOI: https://doi.org/10.5377/wani.v38i76.13541 http://revistas.bicu.edu.ni/index.php/wani wani@bicu.edu.ni

Gadea, V. E. (2002). Estudio Biológico-pesquero de las especies comerciales en San Carlos y Solentiname. ACRA. Río San Juan 2001-2002.

Gassman, J., Rojas, H. L., \& Padron, D. (2017). Reproducción de los róbalos Centropomus undecimalis y C. ensiferus (Perciformes: Centropomidae) en una laguna costera tropical. Revista de Biologia Tropical, 65(1), 181-194.

Gonzalez-Aleman, N. (2006). Ictiofauna del rio Mahogany y su afluente Caño Negro. Bluefields, Nicaragua. Marzo, 2006.

Granado, C. (1996). Ecología de Peces. Universidad de Sevilla. España, Sevilla 2002.

Hossain, M. Y., Arefin, M. S., Mohmud, M. S., Hossain, M. I., Jewel, M. A. S., Rahman, M. M., Ahamed, F., Ahmed, Z. F., \& Ohtomi, J. (2013). Length-weight relationships, condition factor, gonadosomatic index-based size at first sexual maturity, spawning season and fecundity of Aspidoparia morar (Cyprinidae) in the Jamuna River (Brahmaputra River distributary), northern Bangladesh. Journal of Applied Ichthyology, 29(5), 1166-1169.

Hurlbert, S. H. (1970). The nonconcept of species diversity: a critique and alternative parameters. Ecology, 52(4), 342-345.

Le Cren, E. D. (1951). The Length-Weight Relationship and Seasonal Cycle in Gonad Weight and Condition in the Perch ( Perca fluviatilis ). British Ecological Society, 20(2), 201-219.

Lima, L. T. B. de, Oliveira, M. R., Nobrega, M. F., Carvalho, M. M. de, Chellappa, S., \& Oliveira, J. E. L. (2016). Biologia reprodutiva de Bagre marinus (Mitchill , 1815) (Siluriformes: Ariidae) das águas costeiras do Rio Grande do Norte , Brasil. Biota Amazonica, 6(4), 81-86.

Lizama, M. de los A. P., \& Ambrósio, A. M. (2002). Condition factor in nine species of fish of the Characidae family in the upper Paraná River floodplain, Brazil. Brazilian Journal of Biology, 62(1), $113-124$.

Lorán-Núñez, R. M., Martínez-Isunza, F. R., Valdez-Guzmán, A. J., Garduño-Dionate, M., \& MartínezLorán, R. E. (2012). Reproducción y madurez sexual de robalo prieto (Centropomus poeyi) y robalo blanco (C. undecimalis) en el Sistema Lagunar de Alvarado, Veracruz (2005-2007). Ciencia Pesquera, 20, 49-64.

Maldonado-García, M., Gracia-López, V., Carrillo, M., Hernández-Herrera, A., \& Rodríguez-Jaramillo, C. (2005). Stages of gonad development during the reproductive cycle of the blackfin snook, Centropomus medius Günther. Aquaculture Research, 36(6), 554-563.

Margalef, R. (1958). Information theory in ecology. General Systems Bulletin 3: 36-71, University of Louisville. Systems Science Institute, Louisville, Kentucky, 52(4), 35.

Martínez-Morales, I., José Oliva-Paterna, F., Verdiell-Cubedo, D., \& Torralva, M. (2010). Inventario y estado de conservación de la fauna piscícola en la cuenca alta del río Segura (SE Península Ibérica). Anales de Biología, 32, 47-58.

Mendo, J., Samamé, M., Wosnitza-Mendo, C., Mendieta, A., \& Castillo, J. (1988). Analsis biologico pesquero y poblacional de la cachema (Cynoscion analis) del area de Paita, Peru. En Boletin Instituto del Mar del Peru (Vol. 12, Número 2).

Mendoza-Carranza, M., Romero-Rodríguez, A., Segura-Berttolini, E., Ramírez-Mosqueda, E., \& Arévalo(C) 2022 - Bluefields Indian \& Caribbean University (BICU)

Esta obra está bajo una Licencia Creative Commons-Atribución-No Comercial-No Derivada|99 


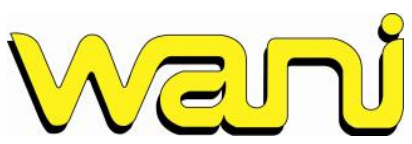

Revista del Caribe Nicaragüense

Núm. 76 | enero - junio, 2022
e-ISSN 2308-7862

Bluefields Indian \& Caribbean University (BICU)

DOI: https://doi.org/10.5377/wani.v38i76.13541 http://revistas.bicu.edu.ni/index.php/wani wani@bicu.edu.ni

Frías, W. (2012). El bagre bandera Bagre marinus como especie clave de la pesca marina de pequeña escala en la costa de Tabasco. Recursos Acuáticos Costeros del Sureste: Tendencias actuales en investigación y estado del arte. RECORECOS, CONCYTEY, UNACAR, UJAT, ECOSUR, UNAM.

MIKUPIA, \& MARENA. (1997). Perfil de los Asuntos de Manejo de las Laguna de Karatá y Wounhta en la Zona Costera de la Región Autónoma del Atlántico Norte de Nicaragua. Bilwi, RACCN, Nicaragua 1997.

Pauly, D. (1983). Some simple methods for the assessment of tropical fish stocks. En FAO Fisheries Technical Paper No. 234 (Número 234).

Perera-Garcia, M. A., Mendoza-Carranza, M., Contreras-Sanchez, W. M., Huerta-Ortíz, M., \& PérezSánchez, E. (2011). Reproductive biology of common snook Centropomus undecimalis (Perciformes: Centropomidae) in two tropical habitats. Revista de Biologia Tropical, 59(2), 669-681.

Pinheiro, P., Broadhurst, M. K., Hazin, F. H. V., Bezerra, T., \& Hamilton, S. (2006). Reproduction in Bagre marinus (Ariidae) off Pernambuco, northeastern Brazil. Journal of Applied Ichthyology, 22(3), 189192.

Ricker, W. (1975). Computation and interpretation of biological statistics of fi sh populations. Board of Canada. 191: 382 pp. Bulletin Fisheries Research, 191, 382.

Roberson, D. R., \& Allen, G. R. (2006). Shorefish of the Tropical Eastern Pacific: an Information System. Ver. 20. En Smithsonian Tropical Research Institute, Balboa, Panamá.

Robins, C. R., Ray, G. C., Douglass, J., Freund, R., National Audubon Society., \& N. W. F. (1986). A field guide to Atlantic Coast fishes of North America. Boston: Houghton Mifflin.

Sanz-Ronda, F. J., Fuentes-Pérez, J. F., \& González, V. S. (2013). Desarrollo de un programa informático para la estimación de parámetros poblacionales de peces en ríos (DimP 1.0). $6^{\circ}$ Congreso Forestal Español. Vitoria-Gasteiz, España, Junio de 2013.

Segura-Berttolini, E. C., \& Mendoza-Carranza, M. (2013). La importancia de los machos del bagre bandera, Bagre marinus (Pisces: Ariidae), en el proceso reproductivo. Ciencias Marinas, 39(1), 29-39.

Shannon, C., \& Weaver, W. (1949). The Mathmatical Theory of Information. Urbana: University of Illinois Press.

Smith, C. L. (1971). A revision of the American groupers: Epinephelus and allied genera. Bulletin of the $A M N H, 146(2)$.

Spellerberg, I. F., \& Fedor, P. J. (2003). A tribute to Claude Shannon (1916-2001) and a plea for more rigorous use of species richness, species diversity and the 'Shannon-Wiener' Index. Global Ecology and Biogeography, 12(3), 177-179.

Taylor, R. G., Whittington, J. A., Grier, H. J., \& Crabtree, R. E. (2000). Age, growth, maturation, and protandric sex reversal in common snook, Centropomus undecimalis, from the east and west coasts of South Florida. Fishery Bulletin, 98(3), 612.

Vergara-Chen, C. (2014). Los robalos (Pisces, Centropomidae) del pacifico de Panama: desafios emergentes en Investigacion y Conservacion. Tecnociencias, 16(1), 15-40.

Villa, J. (1982). Peces Nicaragüenses de agua dulce. Colección Cultural, Banco de América. Serie (C) 2022 - Bluefields Indian \& Caribbean University (BICU)

Esta obra está bajo una Licencia Creative Commons-Atribución-No Comercial-No Derivada|100 
WerL

Revista del Caribe Nicaragüense

Núm. 76 | enero - junio, 2022
e-ISSN 2308-7862

Bluefields Indian \& Caribbean University (BICU)

DOI: https://doi.org/10.5377/wani.v38i76.13541

http://revistas.bicu.edu.ni/index.php/wani

Geografía y Naturaleza número 3. Managua,Nicaragua. 1982.

Winemiller, K. O., \& Rose, K. A. (1992). Patterns of Life-History Diversification in North American Fishes: implications for Population Regulation. Canadian Journal of Fisheries and Aquatic Sciences, 49(10), 2196-2218.

Wootton, R. J. (1990). Ecology of Teleosteos Fishes. Chapman \& Hall. London., 404.

Yáñez-Arancibia, A., Lara-Domínguez, A. L., Aguirre-León, A., Díaz-Ruiz, S., Amezcua-Linares, F., Flores-Hernández, D., \& Chavance, P. (1985). Ecología de poblaciones de peces dominantes en estuarios tropicales: Factores ambientales que regulan las estrategias biológicas y la producción. Ecología de comunidades de peces en estuarios y lagunas costeras, 311-365. 